\title{
molecules
}

ISSN 1420-3049

www.mdpi.com/journal/molecules

Article

\section{Identification of Fungicidal 2,6-Disubstituted Quinolines with Activity against Candida Biofilms}

\author{
Nicolas Delattin ${ }^{1}$, Dorothée Bardiot ${ }^{2}$, Arnaud Marchand ${ }^{2}$, Patrick Chaltin ${ }^{2,3}$, \\ Katrijn De Brucker ${ }^{1}$, Bruno P. A. Cammue ${ }^{1, *}$ and Karin Thevissen ${ }^{1}$ \\ 1 Centre of Microbial and Plant Genetics, Katholieke Universiteit Leuven, Kasteelpark Arenberg 20, \\ B-3001, Heverlee, Belgium \\ 2 CISTIM Leuven vzw, Minderbroedersstraat 12, B-3000, Leuven, Belgium \\ 3 Centre for Drug Design and Discovery, Minderbroedersstraat 8a, B-3000, Leuven, Belgium \\ * Author to whom correspondence should be addressed; E-Mail: Bruno.cammue@biw.kuleuven.be; \\ Tel.: +32-16-329-682; Fax: +32-16-321-966.
}

Received: 13 September 2012; in revised form: 27 September 2012 / Accepted: 15 October 2012 / Published: 18 October 2012

\begin{abstract}
We have identified two subseries of 2,6-disubstituted quinolines, consisting of 6-amide and 6-urea derivatives, which are characterized by fungicidal activity against Candida albicans with minimal fungicidal concentration (MFC) values $<15 \mu \mathrm{M}$. The 6-amide derivatives displayed the highest fungicidal activity against $C$. albicans, in particular compounds 1, 5 and 6 characterized by MFC values of $6.25-12.5 \mu \mathrm{M}$. Compounds $\mathbf{1}$ and $\mathbf{5}$ of this series displayed fungicidal activity against the emerging pathogen Candida glabrata $(\mathrm{MFC}<50 \mu \mathrm{M})$. The 6-amide derivatives 1, 2, 5, and $\mathbf{6}$ and the 6-urea derivatives 10, 12, 13 and 15 could also eradicate $C$. albicans biofilms. We found that the 6-urea derivatives 10, 13, and 15 induced accumulation of endogenous reactive oxygen species in Candida albicans biofilms.
\end{abstract}

Keywords: antifungal agents; Candida spp.; 2,6-disubstituted quinolines; reactive oxygen species; structure-activity relationships

\section{Introduction}

Pathogenic yeasts from the genus Candida can cause serious infections in humans, particularly in immunocompromised patients and are now recognized as the major agents of hospital acquired 
(nosocomial) infections. Candida is recognized as the fourth most common cause of bloodstream infections in the United States, with a high attributable mortality rate [1]. While Candida albicans remains the most common pathogen, non-albicans Candida species, including C. glabrata and C. krusei, with greater resistance to triazoles are being increasingly isolated [1].

Apart from their existence as free-living or planktonic forms, various yeast and fungal species are known to form biofilms upon contact with various surfaces. Moreover, biofilm formation is thought to be a critical step in the development of clinical infections in general [2]. Biofilms consist of dense layers of microorganisms that are surrounded by a self-produced extracellular polymer matrix, and are resistant to most of the currently used antibiotics [3]. Fungal biofilms, especially those of the pathogen C. albicans, are a cause of infections associated with medical devices like indwelling intravascular catheters [4]. Hence, antifungal compounds that can eradicate Candida biofilms are of great therapeutic potential.

The increase of fungal resistance to standard treatments $[5,6]$ has stimulated the search for new antifungal drugs. When designing or screening for novel antifungal drugs, fungicidal activity is generally preferred over fungistatic activity, since it pinpoints to inhibition of targets that are essential for fungal growth [7] or induction of an active cell death pathway, such as apoptosis [8]. An important asset for a novel antifungal lead compound is its potential activity against fungal biofilms.

This study describes the identification and characterization of a series of fungicidal 2,6-disubstituted quinoline derivatives with antibiofilm activity. Various antifungal compounds that are active against Candida biofilms, like miconazole [9] and amphotericin B [10], induce an increased accumulation of endogenous reactive oxygen species (ROS) in biofilm cells. Therefore, we assessed whether these novel antibiofilm compounds also lead to an increased accumulation of endogenous ROS in C. albicans biofilms.

Apart from the observed activity of quinolines against fungal biofilms stated in this study, following reports document the activity of quinolines for other therapeutic indications. Lanza and coworkers identified substituted 4,6-diaminoquinolines as inhibitors of C5a receptor binding, a protein involved in inflammatory diseases [11]. Furthermore, aminoquinolines are known as antimalarial drugs [12] and chloroquin, a 4-aminoquinoline was shown to exhibit antifungal activity against the opportunistic fungus Penicillium marneffei [13]. Finally, 6-acylamino-2-aminoquinolines were identified as potent MelaninConcentrating Hormone 1 Receptor (MCH1R) antagonists, a potential target to tackle obesity [14].

\section{Results and Discussion}

\subsection{Screening and Hit Identification}

A screening campaign for fungicidal compounds resulted in the identification of a fungicidal 2,6-disubstituted quinoline derivative, namely 4-tert-butyl- $N$-(4-methyl-2-(4-methylpiperazin-1yl)quinolin-6-yl)benzamide (9, Table 1a). This screening campaign, which previously led to the identification of antifungal carbazoles [15], consisted of a two-step procedure. In a first step, approximately 5,000 compounds representing diverse chemical classes were screened for antifungal activity against $C$. albicans, in an antifungal spot assay on $C$. albicans-inoculated agar using a single compound dose $(100 \mu \mathrm{g} / \mathrm{mL})$. Next, fungicidal activity of antifungal hits was assessed using two-fold 
dilution series of the compounds, allowing determination of their minimal fungicidal concentration (MFC) against $C$. albicans in phosphate buffered saline (PBS). The MFC was defined as the minimal compound concentration that results in $99 \%$ cell death of the inoculum as compared to the DMSO control treatment [15]. Compound 9, together with the antifungal carbazoles [15], was among the most potent fungicidal compounds identified in this screening campaign. This compound was characterized by fungicidal activity against $C$. albicans with a MFC value of $50 \mu \mathrm{M}$ (Table 2).

Table 1. Selected 6-amide (a) and 6-urea (b) derivatives of 2,6-disubstituted quinolines.

\begin{tabular}{|cccc|}
\hline Compound & $\mathbf{R}^{1}$ & $\mathbf{X}$ & $\mathbf{R}^{2}$ \\
\hline $\mathbf{1}$ & ethyl & $\mathrm{N}$ & 4-tert-butylphenyl \\
$\mathbf{2}$ & ethyl & $\mathrm{N}$ & 4-butylphenyl \\
$\mathbf{3}$ & 2-pyrimidyl & $\mathrm{N}$ & 4-tert-butylphenyl \\
$\mathbf{4}$ & methyl & $\mathrm{CH}$ & 4-tert-butylphenyl \\
$\mathbf{5}$ & methyl & $\mathrm{N}$ & 4-butylphenyl \\
$\mathbf{6}$ & methyl & $\mathrm{N}$ & 4-tert-butylcyclohexyl \\
$\mathbf{7}$ & methyl & $\mathrm{N}$ & 5-chloro-2-methoxyphenyl \\
$\mathbf{8}$ & methyl & $\mathrm{N}$ & 2,4-dimethoxyphenyl \\
$\mathbf{9}$ & methyl & $\mathrm{N}$ & 4-tert-butylphenyl \\
\hline
\end{tabular}

\begin{tabular}{|cc|}
\hline $\mathbf{1 0}$ & 3-chlorophenyl \\
$\mathbf{1 1}$ & phenyl \\
$\mathbf{1 2}$ & 4-chlorophenyl \\
$\mathbf{1 3}$ & 3,5-dimethylphenyl \\
$\mathbf{1 4}$ & 3,4-dimethylphenyl \\
$\mathbf{1 5}$ & 4-ethylphenyl \\
$\mathbf{1 6}$ & 2-methylphenyl \\
$\mathbf{1 7}$ & 2-chlorophenyl \\
$\mathbf{1 8}$ & 4-bromophenyl \\
\hline
\end{tabular}

\subsection{Structure-Activity Relationship}

To analyze the structural determinants for fungicidal activity against $C$. albicans of this new class of compounds, we assessed the MFC of 18 commercially available derivatives divided into two subseries of 2,6-disubstituted quinolines, consisting of 6-amide derivatives (Table 1a) and 6-urea derivatives 
(Table 1b), and of the reference antifungals amphotericin B (AmB) and miconazole (Mico) (Table 2). Next, we assessed their fungicidal activity against $C$. glabrata, as well as their potential antibiofilm activity in terms of the biofilm eradicating concentration $50\left(\mathrm{BEC}_{50}\right)$, which is the minimal concentration of compound resulting in 50\% killing of the $C$. albicans biofilm cells.

Table 2. Antifungal activity of 2,6-disubstituted quinolines against $C$. albicans, C. glabrata and C. albicans biofilms.

\begin{tabular}{cccc}
\hline Compound & C. albicans MFC $^{[\mathbf{a}]}(\boldsymbol{\mu M})$ & $C_{\text {. glabrata }}$ MFC $^{[\mathbf{a}]}(\boldsymbol{\mu M})$ & $\boldsymbol{C .}_{\text {albicans }}$ BEC $_{5 \mathbf{0}}{ }^{[\mathbf{b}]}(\boldsymbol{\mu M})$ \\
\hline $\mathbf{1}$ & 12.5 & 25 & 25 \\
$\mathbf{3}$ & 25 & $>50$ & 25 \\
$\mathbf{4}$ & $>50$ & $>50$ & $>50$ \\
$\mathbf{5}$ & $>50$ & $>50$ & $>50$ \\
$\mathbf{6}$ & 6.25 & 25 & 25 \\
$\mathbf{7}$ & 12.5 & $>50$ & 25 \\
$\mathbf{8}$ & 50 & $>50$ & $>50$ \\
$\mathbf{9}$ & 50 & $>50$ & $>50$ \\
$\mathbf{1 0}$ & 50 & $>50$ & $>50$ \\
$\mathbf{1 1}$ & 50 & $>50$ & 25 \\
$\mathbf{1 2}$ & $>50$ & $>50$ & $>50$ \\
$\mathbf{1 3}$ & $>50$ & $>50$ & 50 \\
$\mathbf{1 4}$ & $>50$ & $>50$ & 50 \\
$\mathbf{1 5}$ & 50 & $>50$ & $>50$ \\
$\mathbf{1 6}$ & $>50$ & $>50$ & 50 \\
$\mathbf{1 7}$ & $>50$ & $>50$ & $>50$ \\
$\mathbf{1 8}$ & 25 & $>50$ & $>50$ \\
AmB & $>50$ & $>50$ & $>50$ \\
Mico & $>3.25$ & $<12.5$ & $<12.5$ \\
\hline
\end{tabular}

${ }^{[\mathrm{a}]}$ Minimal fungicidal concentration; ${ }^{[\mathrm{b}]}$ minimal concentration that results in $50 \%$ killing of biofilm cells.

Compounds 1, 2, 5, 6 and 17 were the most potent fungicidal derivatives against $C$. albicans $(\mathrm{MFC}=6.25-25 \mu \mathrm{M})$. Together with compound 10, these compounds (except for 17) were able to eradicate $C$. albicans biofilms with a $\mathrm{BEC}_{50}$ value identical to the one obtained with miconazole $\left(\mathrm{BEC}_{50}=25 \mu \mathrm{M}\right)$, whereas compounds 12, 13 and 15 showed moderate antibiofilm activity $\left(\mathrm{BEC}_{50}=50 \mu \mathrm{M}\right)$. Only compounds $\mathbf{1}$ and $\mathbf{5}$ showed fungicidal activity against the emerging pathogen C. glabrata $(\mathrm{MFC}=25 \mu \mathrm{M})$. The early SAR study showed that the antifungal activity against C. albicans was partially driven by the presence of a piperazine moiety on the 2 position of the quinoline scaffold, since compound 9 showed a modest MFC value $(50 \mu \mathrm{M})$ whereas compound 4 (piperidine moiety) did not exhibit any fungicidal activity at a concentration higher than $50 \mu \mathrm{M}$. In addition, the introduction of an aromatic (pyrimidyl) substituent on the piperazine moiety (compound 3) instead of a small methyl group (compound 1) led to a complete loss of both antifungal (C. albicans and $C$. glabrata) and antibiofilm activities. Inversely, antifungal (C. albicans) as well as antibiofilm activities were improved by changing the phenyl ring (compound 9) into a cyclohexyl ring (compound 6). The urea derivatives (Table 1b) were in general not active or less potent than the corresponding amide 
derivatives (Table 1a). Only compounds 10 and 17 showed some antibiofilm $\left(\mathrm{BEC}_{50}=25 \mu \mathrm{M}\right)$ or antifungal activity $(C$. albicans $\mathrm{MFC}=25 \mu \mathrm{M})$, respectively. Note that the above SAR was only based on biological data. We did not use QSAR or another computational algorithm.

\subsection{Accumulation of Endogenous ROS}

Antifungal compounds that are active against $C$. albicans biofilms, like miconazole and amphotericin $\mathrm{B}$, were previously shown to induce an increased accumulation of ROS in biofilm cells $[9,10]$ and planktonic cells [16-18]. To assess whether the fungicidal 2,6-disubstituted quinoline derivatives were also able to induce ROS, we measured the endogenous ROS levels via the fluorescent dye 2',7'-dichlorodihydrofluorescein diacetate (H2DCFDA), after induction by the most potent derivatives on $C$. albicans planktonic and biofilm cells. The most active fungicidal derivatives (compounds 1, 2, 5 and 6) against planktonic C. albicans cells, i.e., with MFC values ranging between 6.25-25 $\mu \mathrm{M}$ did not induce ROS accumulation in planktonic cells upon 1,3 or $5 \mathrm{~h}$ of treatment (data not shown). In contrast, $C$. albicans biofilm cells treated with the most active antibiofilm compounds (compounds $\mathbf{1}$, 5, 6, 10, 13 and 15), i.e., compounds characterized with $\mathrm{BEC}_{50}$ values $\leq 50 \mu \mathrm{M}$, induced endogenous ROS accumulation on biofilm cells after $24 \mathrm{~h}$ incubation (Figure 1). Compounds 10, 13 and 15 were characterized by a high capacity to induce ROS accumulation in biofilm cells as indicated by corrected fluorescence values $(\mathrm{CFV})>3,000$. Compounds $\mathbf{1 0}$ and $\mathbf{1 5}$ can induce ROS to the same extent as AmB $(p>0.05)$. CFV are the fluorescence values of the biofilm cells treated with the antifungal compounds subtracted with the value of the DMSO control. Compounds 1, 5 and $\mathbf{6}$ were characterized by an intermediate ROS-induction capacity in biofilm cells $(1,000<\mathrm{CFV}<3,000)$.

Figure 1. Endogenous ROS induction in C. albicans biofilm cells by the most potent compounds against $C$. albicans biofilms. Corrected fluorescence values (CFV) of ROS accumulation by $16 \mathrm{~h}$ old $C$. albicans biofilm cells after treatment $(24 \mathrm{~h})$ with $50 \mu \mathrm{M}$ of the compounds in PBS with a final DMSO background of 2.5\%. Experiments were performed in duplicate and repeated twice. ${ }^{* *} p<0.01 ; * * * p<0.001$.

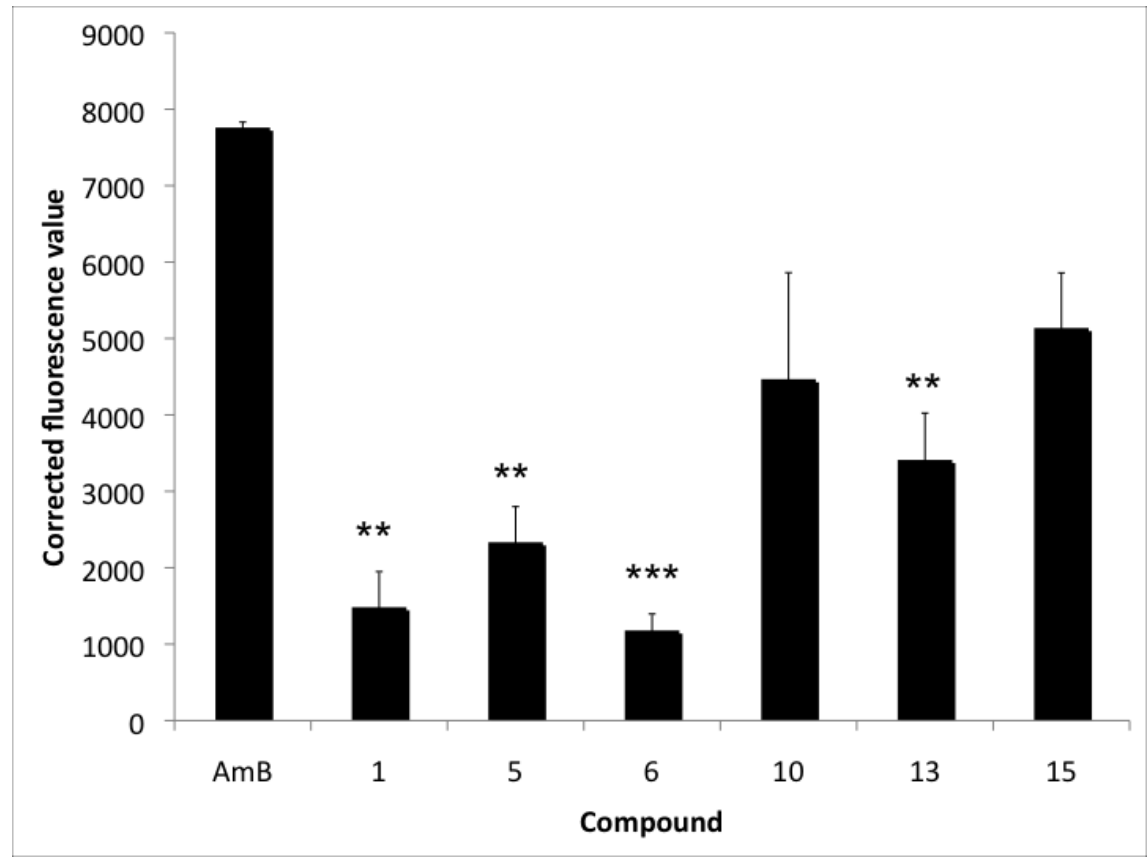




\section{Experimental}

\subsection{Chemical Compounds}

All compounds: 4-tert-butyl- $N$-(2-(4-ethylpiperazin-1-yl)-4-methylquinolin-6-yl)benzamide (1); 4butyl- $N$-(2-(4-ethylpiperazin-1-yl)-4-methylquinolin-6-yl)benzamide (2); 4-tert-butyl- $N$-(4-methyl-2-(4(pyrimidin-2-yl)piperazin-1-yl)quinolin-6-yl)benzamide (3); 4-tert-butyl- $N$-(4-methyl-2-(4-methylpiperidin-1-yl)quinolin-6-yl)benzamide (4); 4-butyl- $N$-(4-methyl-2-(4-methylpiperazin-1-yl)quinolin6-yl)benzamide (5); 4-tert-butyl- $N$-(4-methyl-2-(4-methylpiperazin-1-yl)quinolin-6-yl)cyclohexanecarboxamide (6); 5-chloro-2-methoxy- $N$-(4-methyl-2-(4-methylpiperazin-1-yl)quinolin-6-yl)benzamide (7); 2,4-dimethoxy- $N$-(4-methyl-2-(4-methylpiperazin-1-yl)quinolin-6-yl)benzamide (8); 4-tertbutyl- $N$-(4-methyl-2-(4-methylpiperazin-1-yl)quinolin-6-yl)benzamide (9); 1-(3-chlorophenyl)-3-(4methyl-2-(4-methylpiperazin-1-yl)quinolin-6-yl)urea (10); 1-(4-methyl-2-(4-methylpiperazin-1-yl)quinolin-6-yl)-3-phenylurea (11); 1-(4-chlorophenyl)-3-(4-methyl-2-(4-methyl-piperazin-1-yl)quinolin-6-yl)urea (12); 1-(3,5-dimethylphenyl)-3-(4-methyl-2-(4-methylpiperazin-1-yl)quinolin-6-yl)urea (13); 1-(3,4-dimethylphenyl)-3-(4-methyl-2-(4-methylpiperazin-1-yl)quinolin-6-yl)urea (14); 1-(4ethylphenyl)-3-(4-methyl-2-(4-methylpiperazin-1-yl)quinolin-6-yl)urea (15); 1-(4-methyl-2-(4-methylpiperazin-1-yl)quinolin-6-yl)-3-o-tolylurea (16); 1-(2-chlorophenyl)-3-(4-methyl-2-(4-methylpiperazin1-yl)quinolin-6-yl)urea (17); 1-(4-bromophenyl)-3-(4-methyl-2-(4-methylpiperazin-1-yl)quinolin-6-yl)urea (18) were purchased from ChemDiv (San Diego, CA, USA). Synthesis of the amide derivatives has been described previously [14].

\subsection{Strains and Media}

The yeast strains used in this study were Candida albicans strain SC5314 [19] and Candida glabrata strain BG2 [20]. Overnight cultures were grown in YPD (1\% yeast extract, $2 \%$ peptone and 2\% glucose). Biofilms were grown in SC medium (0.8 g/L CSM, complete amino acid supplement mixture, Bio 101 Systems; $6.5 \mathrm{~g} / \mathrm{L}$ YNB, yeast nitrogen base; $20 \mathrm{~g} / \mathrm{L}$ glucose). PBS consists of $8 \mathrm{~g} / \mathrm{L} \mathrm{NaCl}, 0.2 \mathrm{~g} / \mathrm{L}$ $\mathrm{KCL}, 1.44 \mathrm{~g} / \mathrm{L} \mathrm{Na}_{2} \mathrm{HPO}_{4}$ and $0.24 \mathrm{~g} / \mathrm{L} \mathrm{KH}_{2} \mathrm{PO}_{4}(\mathrm{pH}$ 7.4). Reference antimycotics, amphotericin $\mathrm{B}$ and miconazole were purchased from Sigma-Aldrich (St. Louis, MO, USA).

\subsection{Fungicidal Activity}

The fungicidal activity of antifungal compounds against $C$. albicans and C. glabrata was determined in PBS and the MFC for each compound was calculated according to the definition of Thevissen and coworkers [15]. To this end, overnight cultures of C. albicans or C. glabrata in YPD were $1 / 200$ and $1 / 400$ diluted in PBS, respectively, and treated with the compounds or DMSO $(2.5 \%$ as solvent control) for $24 \mathrm{~h}$ at $37{ }^{\circ} \mathrm{C}$. After $24 \mathrm{~h}$, the MFC was calculated by counting the number of colony forming units (CFUs) as described previously [21].

\subsection{Antibiofilm Activity}

The activity of the compounds against 16 h-old C. albicans SC5314 biofilms was determined in PBS and the $\mathrm{BEC}_{50}$ for each compound was calculated. C. albicans biofilms were grown in sterile 
96-well microtiter plates (TPP, Trasadingen, Switzerland). Overnight cultures of C. albicans in YPD were washed in milliQ water and $1 / 20$ diluted in fresh SC medium. $100 \mu \mathrm{L}$ of this culture was added to each well of a round-bottomed polystyrene 96-well microtiter plate. Following $1 \mathrm{~h}$ of adhesion at $37^{\circ} \mathrm{C}$, the supernatant was removed, the wells were rinsed using milliQ water and incubated with $100 \mu \mathrm{L}$ of fresh SC medium for a growth phase of $16 \mathrm{~h}$. After $16 \mathrm{~h}$ of biofilm formation at $37{ }^{\circ} \mathrm{C}$, wells were rinced with PBS and incubated with $100 \mu \mathrm{L}$ of the antifungal compounds or DMSO $(2.5 \%)$ in PBS at $37^{\circ} \mathrm{C}$. After $24 \mathrm{~h}$ of incubation, biofilms were washed and resuspended in PBS by vigorous vortexing. The $\mathrm{BEC}_{50}$ was determined by counting the number of colony forming units (CFU) as described previously [21].

\subsection{Measurement of ROS Accumulation}

Endogenous amounts of ROS, after treatment of planktonic and biofilm cells with the compounds (2.5\% DMSO) were measured by a fluorometric assay with $2^{\prime}, 7^{\prime}$-dichlorodihydrofluorescein diacetate (H2DCFDA; Molecular Probes, Inc., Eugene, OR, USA) [18,22]. Fluorescence was measured using a fluorescence spectrometer as described [18] and fluorescence values of the samples were corrected by subtracting the fluorescence values of the antifungal compounds with the value of the DMSO control.

\section{Conclusions}

In this study we identified a new series of fungicidal 2,6-disubstituted quinoline derivatives. Among these derivatives, compounds $1,2,5,6$ and 17 were characterized by the highest fungicidal activity against planktonic cultures of $C$. albicans. Moreover compounds $\mathbf{1}$ and $\mathbf{5}$ also showed activity against the emerging pathogen C. glabrata. Furthermore, compounds 1, 2, 5, 6 and 10 showed the most potent activity against $C$. albicans biofilms, with $\mathrm{BEC}_{50}$ values comparable to the one obtained with miconazole $\left(\mathrm{BEC}_{50}=25 \mu \mathrm{M}\right)$. There are several reports documenting the involvement of ROS in the fungicidal activity of compounds against $C$. albicans biofilms, as in case of miconazole and amphotericin B [9,10]. Compounds 10, 13 and 15 showed a high capacity to induce ROS accumulation in C. albicans biofilm cells. Whether the induction of ROS accumulation is the main mechanism of the antibiofilm activity of these new compounds still needs to be investigated. To our knowledge this is the first report regarding the fungicidal activity of these 2,6-disubstituted quinoline derivatives.

\section{Acknowledgments}

N.D. and K.T. acknowledge the receipt of a predoctoral fellowship from IWT-Vlaanderen and a postdoctoral fellowship from the Industrial Research Fund Krediet aan navorsers 1.5.141.09N (K.U.Leuven), respectively. Furthermore, the research leading to these results has received funding from the European Commission's Seventh Framework Programme (FP7/2007-2013) under the grant agreement COATIM (project $n^{\circ} 278425$ ). We want to thank Els Meert for technical assistance. 


\section{References}

1. Marr, K.A. Invasive Candida infections: The changing epidemiology. Oncology (Williston Park) 2004, 18, 9-14.

2. Fanning, S.; Mitchell, A.P. Fungal biofilms. PLoS Pathog. 2012, 8, e1002585.

3. Ramage, G.; Mowat, E.; Jones, B.; Williams, C.; Lopez-Ribot, J. Our current understanding of fungal biofilms. Crit. Rev. Microbiol. 2009, 35, 340-355.

4. Kuhn, D.M.; Ghannoum, M.A. Candida biofilms: Antifungal resistance and emerging therapeutic options. Curr. Opin. Investig. Drugs 2004, 5, 186-197.

5. Klepser, M.E. Candida resistance and its clinical relevance. Pharmacotherapy 2006, 26, 68S-75S.

6. Chamilos, G.; Kontoyiannis, D.P. Update on antifungal drug resistance mechanisms of Aspergillus fumigatus. Drug Resist. Updat. 2005, 8, 344-358.

7. Odds, F.C. Genomics, molecular targets and the discovery of antifungal drugs. Rev. Iberoam. Micol. 2005, 22, 229-237.

8. Thevissen, K.; Hillaert, U.; Meert, E.M.; Chow, K.K.; Cammue, B.P.; Van Calenbergh, S.; Francois, I.E. Fungicidal activity of truncated analogues of dihydrosphingosine. Bioorg. Med. Chem. Lett. 2008, 18, 3728-3730.

9. Vandenbosch, D.; Braeckmans, K.; Nelis, H.J.; Coenye, T. Fungicidal activity of miconazole against Candida spp. biofilms. J. Antimicrob. Chemother. 2010, 65, 694-700.

10. Al-Dhaheri, R.S.; Douglas, L.J. Apoptosis in Candida biofilms exposed to amphotericin B. J. Med. Microbiol. 2010, 59, 149-157.

11. Lanza, T.J.; Durette, P.L.; Rollins, T.; Siciliano, S.; Cianciarulo, D.N.; Kobayashi, S.V.; Caldwell, C.G.; Springer, M.S.; Hagmann, W.K. Substituted 4,6-diaminoquinolines as inhibitors of C5a receptor binding. J. Med. Chem. 1992, 35, 252-258.

12. Foley, M.; Tilley, L. Quinoline antimalarials: mechanisms of action and resistance and prospects for new agents. Pharmacol. Ther. 1998, 79, 55-87.

13. Taramelli, D.; Tognazioli, C.; Ravagnani, F.; Leopardi, O.; Giannulis, G.; Boelaert, J.R. Inhibition of intramacrophage growth of Penicillium marneffei by 4-aminoquinolines. Antimicrob. Agents Chemother. 2001, 45, 1450-1455.

14. Ulven, T.; Frimurer, T.M.; Receveur, J.M.; Little, P.B.; Rist, O.; Norregaard, P.K.; Hogberg, T. 6-Acylamino-2-aminoquinolines as potent melanin-concentrating hormone 1 receptor antagonists. Identification, structure-activity relationship, and investigation of binding mode. J. Med. Chem. 2005, 48, 5684-5697.

15. Thevissen, K.; Marchand, A.; Chaltin, P.; Meert, E.M.; Cammue, B.P. Antifungal carbazoles. Curr. Med. Chem. 2009, 16, 2205-2211.

16. Kobayashi, D.; Kondo, K.; Uehara, N.; Otokozawa, S.; Tsuji, N.; Yagihashi, A.; Watanabe, N. Endogenous reactive oxygen species is an important mediator of miconazole antifungal effect. Antimicrob. Agents Chemother. 2002, 46, 3113-3117.

17. Thevissen, K.; Ayscough, K.R.; Aerts, A.M.; Du, W.; De Brucker, K.; Meert, E.M.; Ausma, J.; Borgers, M.; Cammue, B.P.; Francois, I.E. Miconazole induces changes in actin cytoskeleton prior to reactive oxygen species induction in yeast. J. Biol. Chem. 2007, 282, 21592-21597. 
18. Francois, I.E.; Thevissen, K.; Pellens, K.; Meert, E.M.; Heeres, J.; Freyne, E.; Coesemans, E.; Viellevoye, M.; Deroose, F.; Martinez Gonzalez, S.; et al. Design and synthesis of a series of piperazine-1-carboxamidine derivatives with antifungal activity resulting from accumulation of endogenous reactive oxygen species. ChemMedChem 2009, 4, 1714-1721.

19. Fonzi, W.A.; Irwin, M.Y. Isogenic strain construction and gene mapping in Candida albicans. Genetics 1993, 134, 717-728.

20. Kaur, R.; Ma, B.; Cormack, B.P. A family of glycosylphosphatidylinositol-linked aspartyl proteases is required for virulence of Candida glabrata. Proc. Natl. Acad. Sci. USA 2007, 104, 7628-7633.

21. Aerts, A.M.; Carmona-Gutierrez, D.; Lefevre, S.; Govaert, G.; Francois, I.E.; Madeo, F.; Santos, R.; Cammue, B.P.; Thevissen, K. The antifungal plant defensin RsAFP2 from radish induces apoptosis in a metacaspase independent way in Candida albicans. FEBS Lett. 2009, 583, 2513-2516.

22. Bink, A.; Vandenbosch, D.; Coenye, T.; Nelis, H.; Cammue, B.P.; Thevissen, K. Superoxide dismutases are involved in Candida albicans biofilm persistence against miconazole. Antimicrob. Agents Chemother. 2011, 55, 4033-4037.

Sample Availability: Not available.

(C) 2012 by the authors; licensee MDPI, Basel, Switzerland. This article is an open access article distributed under the terms and conditions of the Creative Commons Attribution license (http://creativecommons.org/licenses/by/3.0/). 